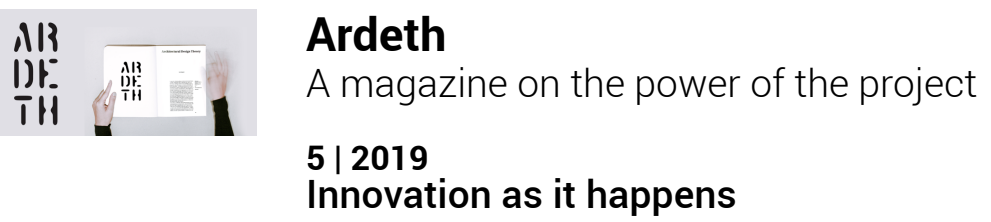

\title{
Innovazione: quale miglioramento?
}

Pratiche di costruzione sociale

Innovation: What Kind of Improvement? Social Construction Practices

\section{Micol Rispoli}

\section{CpenEdition}

\section{Journals}

Edizione digitale

URL: http://journals.openedition.org/ardeth/325

ISSN: 2611-934X

\section{Editore}

Rosenberg \& Sellier

\section{Edizione cartacea}

Data di pubblicazione: 1 novembre 2019

Paginazione: $42-55$

ISSN: 2532-6457

Notizia bibliografica digitale

Micol Rispoli, « Innovazione: quale miglioramento? », Ardeth [Online], 5 | 2019, online dal 01 mai 2020, consultato il 18 novembre 2020. URL : http://journals.openedition.org/ardeth/325 


\section{Innovazione: \\ quale miglioramento? \\ Pratiche di \\ costruzione sociale}

Micol Rispoli

Abstract / Innovation: What Kind of Improvement?

Social Construction Practices.

The term innovation is commonly considered synonymous with improvement. This particularly happens in liberal societies, whose economic dynamics are expressed both in the production of objects and in the design of new services. This meaning in fact has emerged in a productive and social context in which high technology is able to produce increasingly sophisticated instruments and devices that, although requiring a very high level of scientific knowledge, are offered for mass consumption. Moreover, most of the users can only partially understand their functioning. Today innovation seems to be a permanent imperative both at the level of companies and industries, and at the level of institutions, that widely use this term when reforming their services. However, even though the term is mostly used to feed the vicious circle of consumerism, it could also refer to shared design processes and practices capable of promoting unprecedented methods of social construction.

\author{
Affiliation \\ Università degli \\ Studi di Napoli \\ Federico II, \\ Dipartimento di \\ Studi Umanistici - \\ Sezione di Filosofiaa \\ Contacts: \\ micol [dot] rispoli \\ [at] unina [dot] it \\ Received: \\ 27 February 2019 \\ Accepted: \\ 04 September 2019 \\ DOI: \\ 10.17454/ARDETH05.04 \\ ARDETH\#05
}


L'innovazione come carattere dello spirito moderno Il nesso tra i termini innovazione e modernità non ha origini recenti. Da sempre il nuovo ha esercitato il fascino del rivolgimento: rerum novarum cupidus era, nella lingua latina, il rivoluzionario; portare a zero le lancette dell'orologio della storia è stato ricorrente nelle rivoluzioni, basti pensare alle date inaugurali dei calendari introdotti dopo eventi straordinari religiosi o civili, come la nascita di Cristo, la rivoluzione francese, l'inizio dell'era fascista. Nella sua essenza la modernità rappresenta, nell'opinione comune, una rottura con il passato e con le tradizioni che le società pre-moderne ponevano a fondamento della legittimità della loro visione del mondo. Esse infatti si fondavano su grandi racconti (grands récits) mitici che, rendendo sacra la tradizione, garantivano una certa stabilità sociale attraverso l'effetto di coesione prodotto dalla condivisione di uno stesso immaginario.

La condizione moderna si è affermata attraverso un progressivo smantellamento di questi racconti e la loro sostituzione con una razionalità di tipo economico, che ottimizza il rapporto tra mezzi disponibili e fini che si perseguono. $\mathrm{E}$ lo ha fatto proprio quando, in corrispondenza del grande sviluppo della scienza nella prima metà del XVII secolo, la rottura con il passato nasceva dalla volontà di affermare verità esatte e dimostrabili attraverso il metodo e la sperimentazione. Privilegiando poi il rapporto presente/futuro rispetto a quello presente/passato, il termine innovazione si è sempre più legato a quello di progresso che, insieme

Privilegiando poi il rapporto presente/futuro rispetto a quello presente/passato, il termine innovazione si è sempre più legato a quello di progresso. ai termini di futuro e di storia, è uno dei pilastri di quella che chiamiamo condizione moderna.

Oggi, dunque, il termine innovazione ha assunto il significato di sviluppo e miglioramento derivanti dall'avanzamento scientifico. In questa prospettiva si afferma l'idea che la conoscenza scientifica sia di per se stessa fattore di salute, sicurezza, comfort, prosperità e felicità: in una parola, di progresso. In realtà innovazione e progresso sono storicamente termini dal significato diverso e, per di più, il primo non determina necessariamente il secondo.

Fu Saint-Simon (1760-1825) a formulare per primo una vera e propria dottrina del progressismo, che affidava all'industrialismo un ruolo fondamentale per realizzare gli ideali della Rivoluzione francese. Gli autori che fanno riferimento a Saint-Simon, e più 
in generale quelli che, nello sguardo del socialismo scientifico di Marx, furono definiti - con una punta di disprezzo - socialisti utopisti (Owen, Fourier, Cabet, ecc.), condividono una visione dell'industria che ripone grande fiducia nell'organizzazione razionale della produzione. Per Saint-Simon la crescita dell'industria - che considerava autentica istituzione moderna - era il motore di una nuova società, capace di sviluppare una politica favorevole all'interesse pubblico e alla pace sociale, con la promessa del miglioramento continuo del tenore di vita collettivo.

In realtà molte caratteristiche unirebbero le idee di progresso e innovazione: entrambe mettono in questione certezze, valori ereditati e tradizioni - segnalando una rottura con il passato - e mirano a integrare la conoscenza scientifica in un processo di sviluppo che promette una trasformazione migliorativa del benessere collettivo. In questa chiave, innovare assume i significati di razionalizzare e modernizzare e vede nella Ragione - come avvenne con la Rivoluzione francese - la nuova Religione su cui fondare il cammino della civiltà.

Oggi, però, l'innovazione corrisponde a una visione del cambiamento priva della presa e della forza di organizzazione sociale che aveva prima: a venir meno è, nella visione contemporanea, il riconoscimento della conoscenza scientifica come fattore di miglioramento progressivo della condizione umana. La convinzione nel progresso della condizione moderna ha affidato alla conoscenza scientifica il ruolo di presupposto su cui realizzare un futuro di emancipazione. Così la scienza ha assunto un valore e una forza quasi religiose e ha giocato per la psiche moderna lo stesso ruolo della fede per la mentalità pre-moderna: quello di assegnare un senso al divenire umano.

Sostituire, come accade oggi, l'innovazione al progresso, significa non comprenderne la differenza. Mentre le scienze di base vanno perdendo il loro valore originario, la tecnologia si impone sempre più come una vera e propria visione del mondo, sulla base di due false convinzioni: la capacità di miglioramento continuo e incessante e la sua neutralità. Nelle società ad alta tecnologia si ritiene che gli strumenti/oggetti messi a disposizione dei consumatori siano neutrali o oggettivi: i designer si adoperano perché la loro adozione appaia ovvia sotto il profilo logico, accreditando

\section{In realtà molte caratteristiche unirebbero le idee di progresso e innovazione.}




\section{Ci troviamo di} fronte a una sorta di processo di modernizzazione permanente che si svolge però nel quadro di un paradigma "postprogressivo": un miglioramento scientifico e tecnologico sviluppato senza una visione del mondo e senza alcuna ambizione politica. un approccio oggettivistico del fatto tecnico. In realtà, strumenti, macchine e dispositivi che compongono il fenomeno tecnico rinviano a usi costruiti socialmente e che andrebbero considerati nella loro globalità, all'interno di costumi e convenzioni sociali. L'approccio oggettivistico ritiene che le invenzioni tecniche, generate dalle scoperte scientifiche, permettano di risolvere i dilemmi vissuti dall'umanità o che possano semplificare radicalmente la vita.

Nel divenire una vera e propria visione del mondo, la tecnologia ha coinvolto la conoscenza scientifica nell'ingiunzione permanente a innovare. Attribuendo alla coppia scienza e tecnologia il ruolo fondamentale per l'intero funzionamento economico industriale, per legare intimamente la produzione al consumo ininterrotto di oggetti e servizi correlati, l'innovazione programma la loro obsolescenza e stimola incessantemente il loro rinnovamento attraverso il marketing, che nello stesso tempo suscita e modella i desideri dei consumatori. Ci troviamo di fronte a una sorta di processo di modernizzazione permanente che si svolge però nel quadro di un paradigma "post-progressivo": un miglioramento scientifico e tecnologico sviluppato senza una visione del mondo e senza alcuna ambizione politica, creando in tal modo le condizioni più favorevoli al mantenimento e allo sviluppo delle logiche capitalistiche.

\section{L'alienazione dal mondo}

Hannah Arendt propose il concetto originale di alienazione dal mondo (Arendt, 1954) per indicare la posizione dell'uomo moderno che, soggiogato da una profonda convinzione nelle verità scientifiche, preferisce i calcoli razionali alle evidenze sensibili che costituiscono la base del semplice buon senso, $\mathrm{i}$ cui insegnamenti consentono alle persone di resistere alle pressioni dottrinali che alimentano i totalitarismi. Perciò la Arendt sosteneva che scienza moderna e filosofia non solo non erano state in grado di contrastare l'ascesa della barbarie politica del XX secolo, ma le avevano in qualche modo fornito le condizioni di possibilità quantomeno psicologiche. Non solo, ma questa alienazione di cui è vittima l'uomo moderno è sempre in crescita. L'iscrizione del paradigma dell'innovazione nella prospettiva moderna e il riconoscimento del suo carattere scientifico e tecnico amplificano questo 
fenomeno: adottando l'innovazione, intesa in questo senso, come motore economico e sociale, l'alienazione dal mondo è destinata a crescere.

Allo stesso modo l'orizzonte del rinnovamento permanente di prodotti e servizi basati sul desiderio del consumatore rende vana ogni certezza di previsione e, in una condizione dominata da questo paradigma, gli uomini rischiano di diventare estranei al loro stesso divenire. Essere attori del proprio divenire significa provare, per quanto possibile, a dirigerne il corso e assumersene la responsabilità.

\section{Ridare un senso al divenire}

Il modello di sviluppo indotto dalla nozione di innovazione si basa sul carattere più radicale del concetto di progresso: il distacco dalla tradizione con la forza del sapere. Questa volta però senza riprenderne la spinta sociale e morale. Sostituendosi al progresso nelle economie dei paesi sviluppati, l'innovazione ha davvero rinunciato a quella speranza che le aveva dato valore nella religione laica del modernismo? 0 forse c'è un altro modo di chiedersi cosa sia davvero un'innovazione migliorativa? E chi dovrebbe affrontare questa domanda, gli utenti-consumatori, i politici, i filosofi e gli scienziati sociali? In ultimo, può il concetto di innovazione essere inteso al di là o al di fuori del capitalismo? E in che modo?

Oggi sembra interessante immaginare un modello di attività creativa che si collochi oltre la concezione innovativa intesa come miglioramento di un prodotto o servizio a fini commerciali e che possa contribuire a costruire comunità dagli interessi, forme di azione e obiettivi spesso assai eterogenei: ingegneri e industriali, artisti, designer, attori del mondo digitale, gruppi di utenti, cittadini, ecc. In questo sguardo si può interpretare l'innovazione in senso politico.

Certo, la genesi dell'innovazione sfugge in gran parte ai modi gerarchici e pianificati tipici delle organizzazioni ereditate dalla modernità; ma poiché riesce a mettere insieme attori molto differenti dagli interessi eterogenei, con diverse modalità di intervento e obiettivi inizialmente divergenti, non si presenta più come antipolitica, né come a-politica. Al contrario: la gestione di un progetto innovativo permette di sperimentare nuove forme di socialità (...) che assumono valore dal punto di vista politico; in tal senso favorisce la
Sostituire, come accade oggi, l'innovazione al progresso, significa non comprenderne la differenza. 
reinvenzione dell'interesse generale. Anche se essa riguarda oggi, in modo ancora assai parziale, solo le modalità di gestione dell'innovazione, ci lascia intravedere la possibilità di una civiltà nuova a venire (Ménissier, 2016: 56-57).

Innovazione/partecipazione - Innovazione/condivisione Questo orizzonte, che amplia la latitudine dello sguardo del progetto, ha trovato già in passato alcune forme di interrogazione critica. Per quanto riguarda l'architettura è nota quella sviluppata da Giancarlo De Carlo, attraverso l'idea e la pratica della partecipazione. Per De Carlo

il fuoco del ragionamento (...) è la questione culturale: l'architettura come questione culturale. (...) Perché l'architettura sia partecipata, occorre non tanto che la gente sia

Perché

l'architettura sia partecipata, occorre non tanto che la gente sia coinvolta nella sua realizzazione, ma che sia partecipe della sua costruzione coinvolta nella sua realizzazione, ma che sia partecipe della sua costruzione, non in senso tettonico, ma in senso culturale. Occorre che la società si appropri dell'architettura, la faccia propria, si occupi della costruzione di un suo spazio di esistenza (Marini, 2013: 34).

Si tratta di una prospettiva che trova nella riflessione contemporanea sviluppi che vanno ben oltre le forme partecipative alle quali ci si è appena riferiti. Bruno Latour, nel saggio Nessuna innovazione senza rappresentanza! Un parlamento delle cose per i nuovi esperimenti socio scientifici, affronta esplicitamente il tema di quelli che definisce esperimenti collettivi.

Che cosa sono gli esperimenti collettivi, quelli che potremmo anche chiamare esperimenti socio-tecnici? Si tratta di esperimenti condotti senza regole? Sarebbe auspicabile stabilire delle regole in proposito? Che cosa significa disporre di regole secondo la vecchia definizione di razionalità o di comportamento razionale? E ancora, che cosa ha a che fare tutto ciò con il concetto europeo di democrazia? (Latour, 2006: 67-68).

Su queste domande Latour espone i termini di ciò che sta diventando un movimento di pensiero molto vasto. La sua tesi è che molti di questi esperimenti collettivi sono usciti fuori dai limiti che un tempo li confinavano in laboratori specialistici, per coinvolgere oggi una pluralità di persone. Un tempo la scienza era un'attività praticata dentro luoghi chiusi in cui lavoravano specialisti, mentre fuori cominciava il re- 
gno della pura esperienza empirica, del tutto diverso da quello della sperimentazione scientifica.

Oggi il laboratorio ha in molti casi allargato i suoi confini a tutto il pianeta. I suoi strumenti sono ovunque - basti pensare, ad esempio, al sistema globale di localizzazione, ovvero la rete satellitare che consente oggi di raccogliere dati con lo stesso grado di precisione sia dentro che fuori dai laboratori, o ai sistemi di monitoraggio relativi all'acidificazione delle acque, alle attività dei vulcani e dei ghiacciai - e tengono sotto controllo continuo il mondo esterno.

Inoltre oggi gli esperimenti sono condotti da tutti noi $a$ scala reale e in tempo reale, come è diventato del tutto evidente con il problema drammatico del riscaldamento globale. In questo caso, per quanto siano in corso simulazioni, modellazioni e monitoraggi complessi attraverso potenti calcolatori,

il vero esperimento è condotto su di noi, con noi, attraverso il comportamento di ognuno e di tutti e con la partecipazione degli oceani, della stessa atmosfera e anche della corrente del golfo - come sostengono alcuni geografi. L'unico modo per sapere se il riscaldamento globale è veramente dovuto all'attività antropica è quello di misurare le emissioni nocive che produciamo, di interromperle e di verificare che cosa succede a quel punto sul piano collettivo. Si tratta di un vero esperimento, ma un esperimento a scala reale nel quale siamo tutti coinvolti (Latour, 2006: 72)

Un esperimento senza neppure un proprio protocollo e senza che a nessuno sia esplicitamente assegnata la responsabilità del suo controllo. Chi ha il potere di dire l'ultima parola, di decidere per tutti noi?

Per questo Latour, quando afferma che è scomparsa la distinzione tra interno ed esterno del laboratorio, ci ricorda semplicemente che il dibattito scientifico contemporaneo sta delineando la presenza di forum ibridi (Callon et al., 2001) spazi compositi che mettono insieme sapienti, esperti professionisti della politica, cittadini, autori di dibattiti socio-tecnici (dismissione del nucleare, AIDS, ecc).

Finora sono esistiti soltanto un forum che aveva il compito di rappresentare le cose della natura - dove si parlava di rappresentazione come accuratezza, precisione, competenza - e un forum che aveva il compito di rappresentare gli uomini appartenenti a
Oggi gli
esperimenti sono
condotti da tutti
noi a scala reale
e in tempo reale,
come è diventato
del tutto evidente
con il problema
drammatico del
riscaldamento
globale. 


\section{Le regole di metodo sono diventate regole nuove, non servono per governare il Parco Umano, ma per elaborare insieme il protocollo degli esperimenti collettivi.}

una società - dove si parlava di rappresentanza come affidabilità, fiducia, obbedienza. Oggi i due significati di rappresentanza si sono fusi e un esempio di forum ibrido è proprio il dibattito attuale sul riscaldamento del pianeta.

Intorno a un tavolo stanno seduti i diversi portavoce: alcuni rappresentano l'atmosfera alta, altri le diverse lobbies del petrolio e del gas, altri ancora le organizzazioni non governative, altri ancora rappresentano nel senso proprio del termine, i rispettivi elettori. La netta differenza che prima separava i rappresentanti delle cose e quelli delle persone è semplicemente svanita. Ciò che conta ora è che tutti i portavoce si ritrovano insieme, in una stessa stanza, sono coinvolti nello stesso esperimento collettivo, e discutono nello stesso momento delle interconnessioni che coinvolgono persone e cose (Latour, 2006: 73).

Questo, per Latour, significa - con esplicito riferimento alle tesi sostenute da Peter Sloterdijk (1999a) - che occorre individuare una nuova politica. Con una necessaria premessa:

pensare agli artefatti in termini di progetto significa concepirli sempre meno come oggetti e pensarli sempre più come cose. Gli artefatti stanno diventando concepibili come complessi assemblaggi di questioni contraddittorie - questo è il significato etimologico della parola "cosa”, thing, in inglese, così come in altre lingue europee. Nel momento in cui le cose sono prese in considerazione per verificare se sono bene 0 male progettate, allora esse non appaiono più come materie di fatto ma come materie in questione (Latour, 2009: 265).

Da qui nasce l'espressione che egli utilizza per indicare questa nuova politica, intesa come costituzione di un 'Parlamento delle Cose' (Parliament of Things). Le regole di metodo sono diventate regole nuove, non servono per governare il Parco Umano, ma per elaborare insieme il protocollo degli esperimenti collettivi. Facendo, inoltre, esplicito riferimento a una riflessione di John Dewey, Latour opera un paradossale ribaltamento tra i significati di privato e pubblico nella condizione contemporanea. Per Dewey infatti privato non significa necessariamente individuale 0 soggettivo, ma qualcosa che è ben noto, prevedibile, comunemente accettato. Il pubblico, invece, inizia con 
ciò che non possiamo vedere né prevedere, con le conseguenze impreviste, indesiderate, invisibili delle nostre azioni collettive.

Contrariamente a tutti i sogni di politica razionale che hanno devastato il nostro continente per secoli, Dewey equipara il pubblico non alla superiore conoscenza da parte delle autorità, ma alla loro cecità. Il pubblico nasce quando siamo confusi, quando non sappiamo il perché delle cose, (...) quando non ci sono esperti in grado di determinare le conseguenze dell'azione collettiva (Latour, 2006: 87).

Nasce perciò l'esigenza di assumere quello che Latour definisce principio di precauzione: un principio che non comporta una sospensione di iniziativa, ma che è invece un richiamo alla sperimentazione, all'invenzione, all'esplorazione e, ovviamente, al rischio. Ciò significa che noi dobbiamo oggi trattare i "casi" (matters of concerns) scientifici e tecnici (e cioè gran parte delle grandi questioni contemporanee) con modalità non differenti da quelle con le quali ci occupiamo di tutto ciò che fa parte della nostra vita quotidiana.

Lo stesso vale per quel che riguarda piantare alberi, procreare, custodire denaro, prestarlo, armarci contro potenziali nemici, e così via. Di fronte a tutte queste decisioni, prendere dei rischi e prendere delle precauzioni sono sinonimi: più rischiamo, più dobbiamo essere attenti e vigili. È ciò che chiamiamo esperienza. Lo sviluppo del principio di precauzione significa che ciò che è sempre stato vero nell'esperienza quotidiana, diventa ora vero anche per l'ambito della scienza e della tecnologia: piuttosto che aspettare di essere assolutamente certi prima di fare la minima mossa, sappiamo che dobbiamo sperimentare mantenendo l'equilibrio tra l'audacia e la preoccupazione.

Se, con Dewey, consideriamo che il pubblico non è nelle mani degli specialisti illuminati, è proprio il ruolo dell'esperto - inteso nel modo tradizionale una figura, cioè, che ha l'incarico di mediare fra chi produce conoscenza nel chiuso dei laboratori e il resto della società, che va scomparendo. Negli esperimenti collettivi in cui siamo oggi impegnati questa divisione non esiste più. Michel Callon, Pierre Lascoumes e Yannick Barthe (2001) propongono di sostituire la vecchia nozione di esperto con quella più ampia di co-ricercatore. Siamo portati tutti a formulare problemi di

\section{Dobbiamo oggi trattare i "casi" scientifici e tecnici con modalità non differenti da quelle con le quali ci occupiamo di tutto ciò che fa parte della nostra vita quotidiana.}




\section{Come consumatori, come militanti, come cittadini, siamo tutti co-ricercatori.}

ricerca, sia chi è rinchiuso nel suo laboratorio sia chi è definito da questi autori ricercatore esterno (ovvero tutti noi). Siamo tutti impegnati, a vario titolo, negli esperimenti collettivi su temi diversi come il clima, l'alimentazione, il paesaggio, la salute, la progettazione urbana, la cittadinanza, la comunicazione tecnica, ecc.: come consumatori, come militanti, come cittadini, siamo tutti co-ricercatori.

Vi è ovviamente differenza tra le diverse occupazioni, ma non quella differenza che esiste tra chi produce conoscenza e chi è investito dalle sue applicazioni.

Conclusione: ri-costruire ambiti comunitari.

Crisi economica, crisi ambientale e rivoluzione tecnologica - quella informatica in particolare - stanno determinando, nel loro insieme, una radicale trasformazione di stili di vita, di prospettive e di capacità di immaginare il mondo futuro. Sembra che il sapere progettuale sia divenuto strumento del tutto inadeguato per generare prestazioni ambientali, sociali, economiche e che corrisponda in qualche modo a questa condizione se non liquida, comunque instabile e incerta.

Qual è il destino dell'architettura in queste condizioni? Appare sempre più necessaria una nuova idea di progetto dello spazio fisico. In tale prospettiva il progetto come azione sociale è al centro di molti lavori contemporanei che si interrogano su spazi, attrezzature e infrastrutture per l'abitare, sui quali peraltro le ultime due Biennali di Venezia (2016 e 2018) hanno posto, in diverso modo, l'accento.

Tra le sperimentazioni più interessanti in tal senso va annoverata quella della piattaforma multidisciplinare spagnola Zoohaus, i cui membri, nel 2010, insieme ai residenti del quartiere La Latina, hanno occupato e assunto la gestione di un lotto libero nel centro di Madrid ribattezzato El Campo de Cebada. Grazie a operazioni quali la creazione di un orto comunitario, l'organizzazione di eventi teatrali, sportivi e musicali, l'attivazione di laboratori per bambini, il progetto può essere considerato come un esempio un commons urbani. Nel 2013 El Campo, diventato un modello per gli spazi della comunità in Spagna, ha ricevuto il primo premio dalla Biennale di architettura e urbanistica spagnola. Si tratta di uno dei progetti di infrastruttura open source che invitano a riconsiderare la natura 
della cosa pubblica attraverso processi ricorsivi deliberatamente aperti al riadattamento. (Domínguez Rubio, Fogué, 2013)

Un'altra sperimentazione esemplare è quella della Cooperativa La Borda che, insieme alla Cooperativa di architetti Lacol, ha promosso un modello per fornire abitazioni economiche in regime non speculativo. Per il progetto, iniziato nel 2012, di 32 unità coabitative per 60 inquilini, a Sants (Barcellona), la municipalità ha offerto un terreno, riservandosi il diritto di superficie, e banche legate alla economia sociale hanno favorito l'accesso al credito. Le linee guida sono: la partecipazione degli inquilini all'intero processo, la creazione di strutture flessibili riadattabili in futuro e l'introduzione di iniziative di quartiere. Attraverso workshop ogni famiglia può progettare e lavorare insieme ai tecnici, introducendo spazi produttivi e ambiti comuni per favorire la vita comunitaria e ottimizzare i servizi. Tra gli aspetti più significativi vi è un cambiamento della definizione di domesticità e una trasformazione dei limiti tra spazio pubblico e spazio individuale a favore di una condizione a metà strada tra sfera pubblica e privata.

Questa modalità del progetto investe edifici, quartieri, luoghi pubblici, infrastrutture, ecc. e si realizza attraverso processi di azione progettuale condivisa che producono forme di coesione molto più intense di quelle legate alla semplice partecipazione (almeno nel senso che questo termine ha assunto nelle esperienze precedenti). In queste azioni progettuali il concetto tradizionale di autorialità viene sostituito da pratiche di condivisione del processo creativo che possono contribuire alla creazione di inedite forme comunitarie. Può essere questo un modo per interpretare positivamente la riflessione di Peter Sloterdijk laddove, nel terzo volume della sua trilogia (Sloterdijk, 1998, 1999b, 2004), descrive l'idea di società schiumosa, successiva al definitivo tramonto della macrosfera europea.

Per mezzo del concetto di schiuma, descriviamo degli agglomerati di bolle. (...) Questa espressione designa dei sistemi o degli aggregati di vicinanze sferiche in cui ogni 'cellula' costituisce un contesto auto complementare - in linguaggio corrente: un mondo, un luogo - (...) o ancora un focolare (Haushalt) (...). Ciascuno di questi focolari, ciascuna

\section{Questa modalità del progetto si realizza attraverso processi di azione progettuale condivisa che producono forme di coesione molto più intense di quelle legate alla semplice partecipazione}


È possibile stabilire processi di ricomposizione parziali, aggregati più ampi di microsfere capaci di sviluppare reti di interconnessioni comuni, forme comunitarie più ampie e condivise senza pretese totalizzanti. di queste simbiosi e alleanze è una serra di relazioni (Beziehung-Treibhaus) sui generis (Sloterdijk, 2004: 55).

Dunque ciò che rimane è un agglomerato di microsfere, correlate tra loro in macrosfere minime (del tipo delle comunità religiose o politiche), ma niente che assomigli più al sistema di inclusività totale che era la macrosfera di un tempo. Nelle schiume contemporanee crolla l'interconnessione che la macrosfera occidentale europea (e le macrosfere in genere) dava ai vari momenti di cui i rapporti umani sono composti.

In ogni punto nella schiuma si aprono delle visioni regionali nel limitrofo, ma non si dispone di una visione d'insieme (...) quando parliamo di schiume in questo tono, ci siamo apertamente separati dal simbolo centrale della metafisica classica, la monosfera che riunisce tutto: l'Uno in forma di sfera e la sua proiezione nelle costruzioni centrali panottiche (Sloterdijk, 2004: 62-63).

Rispetto agli estremi della grande, unica sfera metafisica - di cui fortunatamente non avvertiamo più il bisogno - e della dissoluzione nella società liquida annunciata da Bauman (Bauman, 2000), è possibile stabilire processi di ricomposizione parziali, aggregati più ampi di microsfere capaci di sviluppare reti di interconnessioni comuni, forme comunitarie più ampie e condivise senza pretese totalizzanti. Per farlo, piuttosto che pensare a prodotti innovativi, dar luogo a processi, procedure progettuali innovativi che coinvolgono più attori appare sempre di più oggi una strada da intraprendere, se non per scelta, quanto meno per condizione (Sloterdijk, 2009).

\section{Bibliografia}

Akrich, M. et al. (2002), The Key Success in Innovation. The Art of Interessement, Uxbridge, Brunel University.

Arendt, H. (1954), The Human Condition, Chicago, University Chicago Press; ed. it. Vita activa. La condizione umana, Milano, Bompiani, 1989.

Bauman, Z. (2000), Liquid modernity, Cambridge, Polity Press; trad. it. di S. Minucci, Modernità liquida, Roma-Bari, Laterza, 2011.

Callon, M. et al. (2001), Agir dans un monde incertain. Essai sur la démocratie technique, Paris, Le Seuil.

Domínguez Rubio, F., Fogué, U. (2013), Technifying public space and publicizing infrastructures: Exploring new urban political ecologies through the square of General Vara del 
Rey, "International Journal of Urban and Regional Research", vol. 37 .

Innerarity, D. (2006), La démocratie sans l'État. Essai sur le gouvernement des societés complexes, Paris, FlammarionClimats.

Latour, B. (2006), Nessuna innovazione senza rappresentanza! Un parlamento delle cose per i nuovi esperimenti socioscientifici, in M. Bucchi (a cura di), Sapere, Fare, Potere. Verso un'innovazione responsabile, Soveria Mannelli (CZ), Rubettino.

Latour, B. (2009), Un Prometeo cauto? Primi passi verso una filosofia del design, in $\mathrm{E} / \mathrm{C}$ serie speciale, numero monografico dal titolo Il discorso del design. Pratiche di progetto e saper fare semiotico (a cura di D. Mangano, A. Mattozzi) nn. 3/4, 2009.

Marini, S. (2013), Introduzione. Scegliere la parte, in S. Marini, G. De Carlo (a cura di), L'architettura della partecipazione, Macerata, Quodlibet.

Ménissier, T. (2016), Innovation et Histoire. Une critique philosophique, "Quaderni Communication, technologies, pouvoir”, n. 91.

Sloterdijk, P. (1998), Sphären I - Blasen, Mikrosphärologie, Frankfurt a.M., Suhrkamp; trad. it. di G. Bonaiuti (a cura di), Sfere I. Bolle, Roma, Meltemi, 2009.

Sloterdijk, P. (1999a), Regeln für den Menschenpark, Frankfurt a.M., Suhrkamp.

Sloterdijk, P. (1999b), Spharen 2: Globen, Frankfurt a.M., Suhrkamp; trad. it. di G. Bonaiuti (a cura di), Sfere II. Globi, Milano, Raffaello Cortina, 2014.

Sloterdijk, P. (2004) Sphären III - Schäume, Plurale Sphärologie, Frankfurt a.M., Suhrkamp; trad. it. di G. Bonaiuti (a cura di), Sfere III. Schiume, Milano, Raffaello Cortina, 2015.

Sloterdijk, P. (2009), Du mußt dein Leben ändern. Über Religion, Artistik und Anthropotechnik, Frankfurt a.M., Suhrkamp; trad. it. di P. Perticari (a cura di), Devi cambiare la tua vita, Milano, Raffaello Cortina, 2010. 